\title{
Avaliação de conforto térmico em ambientes cirúrgicos utilizando método de Fanger e temperaturas equivalentes
}

\author{
Evaluation of thermal comfort in surgical \\ environments using Fanger's method and \\ equivalent temperatures
}

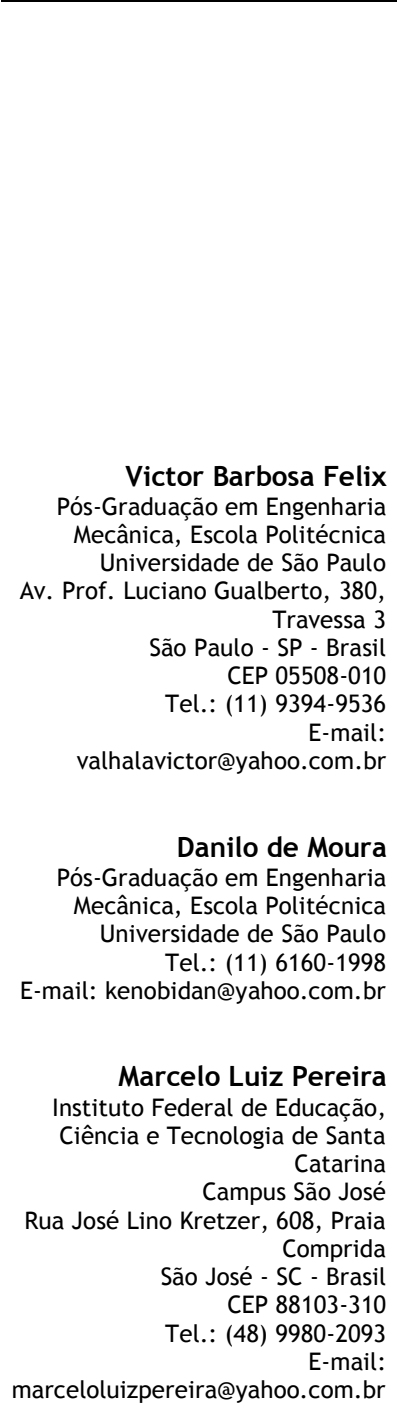

Arlindo Tribess Departamento de Engenharia Mecânica, Escola Politécnica Universidade de São Paulo

Tel.: (11) 3091-9660 E-mail: atribess@usp.br

Recebido em 05/03/10 Aceito em 23/08/10

\section{Victor Barbosa Felix \\ Danilo de Moura \\ Marcelo Luiz Pereira \\ Arlindo Tribess}

\section{Resumo}

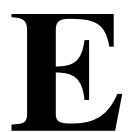

m ambientes cirúrgicos as condições de conforto térmico precisam ser as melhores possíveis para que o cirurgião e a equipe médica trabalhem em ambientes cirúrgicos as condições de conforto térmico precisam ser as melhores possíveis para que o cirurgião e a equipe médica trabalhem em condições favoráveis para o sucesso do procedimento cirúrgico. Neste trabalho são avaliadas condições de conforto térmico em salas cirúrgicas utilizando o método de Fanger e temperaturas equivalentes. Foram realizadas medições de variáveis ambientais e avaliações subjetivas baseadas em questionários. A aplicação do método de Fanger mostrou-se adequada na avaliação de condições de conforto térmico em salas cirúrgicas, embora seja necessário cuidado especial em sua utilização e na análise dos resultados. Paralelamente, resultados de estudo comparativo com trabalhos de outros autores mostraram valores praticamente iguais de temperaturas equivalentes de conforto térmico, em torno de $22 \mathrm{oC}$, para todos os membros da equipe cirúrgica. Esse resultado é particularmente útil para avaliar diferentes condições ambientais e pessoais em propiciar condições adequadas de conforto térmico aos diferentes membros da equipe cirúrgica

Palavras-chave: Conforto térmico. Método de Fanger. Temperaturas equivalentes. Ambientes cirúrgicos

\section{Abstract}

In surgical environments thermal comfort conditions need to be the best possible to allow surgeons and the medical team to work in favourable conditions for the success of procedures. In this study, thermal comfort conditions in surgery rooms were analyzed using Fanger's method and equivalent temperatures.

Measurements of environmental variables and subjective evaluations based on questionnaires were undertaken. The application of Fanger's method was proven to be appropriate for the evaluation of thermal comfort conditions in surgical rooms, although special care is necessary on its use and in the analysis of the results. Moreover, results of a comparative study with other authors' works showed practically the same values of thermal comfort equivalent temperatures, which were around 22 oC for all members of the surgical team. That result is particularly useful to evaluate different environmental and personal conditions in order to provide suitable thermal comfort conditions for different members of the surgical team.

Keywords: Thermal comfort. Fanger's method. Equivalent temperatures. Surgical environments. 


\section{Introdução}

Ao longo da história da humanidade, o homem tem buscado conforto, proteção e segurança nos ambientes onde vive. As características atuais das sociedades, tanto desenvolvidas como em desenvolvimento, fazem com que um elevado número de pessoas passe a maior parte de seu dia em ambientes interiores. Quando esses ambientes não apresentam características adequadas ao uso, podem, muitas vezes, gerar condições adversas, de má qualidade do ar interior, desconforto térmico, problemas de saúde e perda de produtividade.

A qualidade do ar e o conforto térmico em ambientes interiores estão relacionados às características do ambiente e de movimentação do ar, que podem afetar a saúde e o conforto dos ocupantes de uma edificação. Quando os ambientes interiores são de hospitais e demais instalações médicas, é importante uma atenção ainda maior, pois constituem ambientes complexos, que requerem ventilação adequada para o controle de contaminação aérea, o conforto de pacientes, de pessoal e de visitantes.

Em ambientes cirúrgicos, as intervenções cirúrgicas complexas e demoradas que se realizam hoje exigem atividades intensas, uma grande equipe de pessoas e o uso de muitos tipos de equipamentos. Essa situação torna importante o controle eficaz das variáveis que interferem na saúde, conforto e bem-estar do paciente e da equipe cirúrgica (DHARA; PITTET, 2002).

Assim, o principal objetivo dos sistemas de tratamento de ar e de ventilação em ambientes cirúrgicos é diminuir os riscos de infecções, do paciente e dos profissionais de saúde, que podem ser causadas por partículas transportadas pelo ar. Para que isso ocorra de forma adequada, os sistemas de distribuição de ar devem trabalhar com uma ventilação especial, baseada em taxas fixas de geração de contaminantes, diferenciais de pressão, umidade, entre outros parâmetros (HERMANS, 2000).

Simultaneamente, os sistemas de ventilação e de distribuição de ar em salas cirúrgicas devem garantir o conforto térmico do paciente e da equipe cirúrgica durante a cirurgia, o que, na maioria das vezes, fica em segundo plano. Condições térmicas confortáveis ajudam a equipe cirúrgica a trabalhar melhor e previnem possíveis problemas ao paciente. Resultado do sistema de ventilação e tratamento de ar utilizado, as condições de conforto térmico e desconforto local em ambientes internos estão diretamente relacionadas com a forma que o ar se movimenta no interior do ambiente. A movimentação do ar terá um padrão característico do sistema de insuflamento utilizado, com perfis de temperatura e velocidade completamente diferentes daqueles verificados em ambientes com outros tipos de sistemas de ventilação (PEREIRA; TRIBESS, 2005).

Existem poucos estudos de avaliação experimental de conforto térmico em salas cirúrgicas encontrados na literatura, que se restringem basicamente aos trabalhos de Wyon, Lidwell e Willians (1968) e de Mora, English e Athienitis (2001).

Em trabalho pioneiro, Wyon, Lidwell e Willians (1968), na Inglaterra, avaliaram as condições de conforto térmico da equipe médica para servir de orientação no projeto e operação de salas cirúrgicas nas ilhas britânicas. Trata-se de um trabalho bastante extenso, em que foram avaliadas condições de conforto térmico em 25 salas cirúrgicas ao longo das quatro estações do ano. Nas visitas às instalações, foram respondidos questionários, anotados detalhes da vestimenta, sexo, idade, função exercida, tipo de cirurgia e duração, bem como realizadas medições de temperatura, velocidade e umidade do ar, e de temperatura de globo. Não foram fornecidos detalhes do sistema de ventilação. Aparentemente, as avaliações foram feitas em condições de ventilação natural. Os autores verificaram que existe uma diferença significativa entre as condições ambientais para o conforto térmico dos cirurgiões, anestesistas e outros membros da equipe cirúrgica. Ressaltaram, como igualmente importante, que os requisitos para a especificação de um ambiente térmico confortável em salas cirúrgicas diferem daqueles de ambientes de escritórios, ambientes fabris e outros ambientes estudados até então. Isso porque, enquanto os ambientes térmicos convencionais são ocupados por um grande número de pessoas e nos quais se procura obter condições de conforto ao maior número possível dos ocupantes, em salas cirúrgicas o conforto dos cirurgiões deve ser priorizado e o desconforto para os demais deve ser minimizado.

Para a análise dos resultados, Wyon, Lidwell e Willians (1968) definiram um "índice de temperatura" (similar ao da temperatura equivalente) como sendo uma combinação da temperatura do ar, da temperatura radiante média em um ambiente com $50 \%$ de umidade relativa e velocidade do ar de $0,13 \mathrm{~m} / \mathrm{s}$ (25 pés $/ \mathrm{min})$. Os autores encontraram que esse índice de temperatura, com o qual a maioria do pessoal da equipe cirúrgica estaria confortável, seria de 20,5 ${ }^{\circ} \mathrm{C}$ e que, para o maior conforto dos cirurgiões e 
dos anestesistas, deveria ser de $18{ }^{\circ} \mathrm{C}$ e de $21,5^{\circ} \mathrm{C}$ respectivamente.

Mais recentemente, Mora, English e Athienitis (2001) realizaram estudo em duas salas cirúrgicas em um hospital no Canadá. As salas eram providas de sistema convencional de $\mathrm{HVAC}^{1}$ (com 100\% de ar renovado, volume de ar constante (VAC), reaquecimento terminal e um fan-coil dedicado para cada sala cirúrgica), com sistema de distribuição de ar com fluxo unidirecional ("laminar"). Foram realizados oito testes em sala cirúrgica de fluxo unidirecional com painéis, que são proteções colocadas em torno da mesa cirúrgica, com o objetivo de restringir a movimentação do ar para que este não se misturasse com o ar das demais partes da sala, e três testes em sala de fluxo unidirecional com cortina de ar, envolvendo cirurgias ortopédicas.

No trabalho de Mora, English e Athienitis (2001), os testes foram realizados com temperatura média na sala cirúrgica em torno de $21,5{ }^{\circ} \mathrm{C}$, com variações entre $19^{\circ} \mathrm{C}$ e $25^{\circ} \mathrm{C}$, devido a problemas no controle do sistema de HVAC. Em adição aos questionários, o modelo de conforto de Fanger (1972) foi adotado para avaliar as condições de conforto térmico da equipe cirúrgica e do paciente com diferentes tipos de vestimentas.

Mora, English e Athienitis (2001) também verificaram que, em função das condições do ambiente e das condições das pessoas (vestimenta, atividade, posição no ambiente), não é possível prover condições de conforto térmico para todos os membros da equipe cirúrgica. Com base no modelo de Fanger, os autores concluíram que a temperatura do ar deveria ficar em torno de $19^{\circ} \mathrm{C}$ para prover condições de conforto para os cirurgiões. Contudo, nessa temperatura, as enfermeiras e anestesistas deveriam estar com roupas com pelo menos 0,9 clo, e o paciente coberto com pelo menos 1,6 clo.

Os valores de "índice de temperatura" apresentados por Wyon, Lidwell e Willians (1968) foram obtidos por uma análise de regressão que relacionou variáveis ambientais e pessoais com as respostas subjetivas dos membros da equipe cirúrgica com relação a sua sensação térmica. Os autores avaliaram a relação que cada variável analisada tem na influência da resposta das pessoas com relação a sua sensação térmica e definiram esse índice como sendo a temperatura de um ambiente onde pessoas sob condições específicas, tais como a temperatura do ar igual à temperatura radiante média, velocidade do ar em torno de 0,13 $\mathrm{m} / \mathrm{s}$, e umidade relativa do ar em torno de $50 \%$,

${ }^{1}$ HVAC - Heating, Ventilating and Air Conditioning, ou AVAC Aquecimento, Ventilação e Ar Condicionado. deverão manifestar, por meio de votos, a mesma resposta de sensação térmica que manifestarão no ambiente estudado. Embora esse índice englobe todas as variáveis ambientais relacionadas com o conforto térmico, a definição do "índice de temperatura" é particularizada para determinada condição de velocidade e de umidade relativa do ar.

Mora, English e Athienitis (2001), por sua vez, utilizaram a temperatura do ar para referenciar condições ambientais de conforto de cada membro da equipe cirúrgica. Embora a temperatura do ar possa ser considerada como a variável mais importante na sensação térmica experimentada pelas pessoas (ROHLES JÚNIOR, 2007), esse "índice" não considera as demais variáveis ambientais.

Uma forma de se considerarem todas as variáveis ambientais em um único índice é com adoção da temperatura equivalente, que é definida como a temperatura de um ambiente imaginário com temperatura radiante média igual à temperatura do ar e com velocidade do ar igual a zero, onde uma pessoa troca a mesma quantidade de calor por convecção e radiação que trocaria em um ambiente real. $\mathrm{O}$ conceito de temperatura equivalente é bastante utilizado em estudos das condições de conforto térmico em ambientes automotivos, como, por exemplo, na análise de conforto térmico em cabines de aeronaves (MOURA, 2009)

Segundo NILSSON (2004), a vantagem da temperatura equivalente é que ela expressa os efeitos das variáveis térmicas combinadas para uma única pessoa, de forma que a interpretação é simples, sendo particularmente útil para a avaliação de diferentes condições ambientais. Bohm et al. $\left(1990^{2}\right.$ apud NILSSON, 2004) e Schwab, Conrad e Mayer $\left(1999^{3}\right.$ apud NILSSON, 2004) verificaram que, para condições próximas da neutralidade térmica e com variações pequenas das variáveis ambientais, o valor da temperatura equivalente sempre representa a mesma resposta subjetiva, independentemente do tipo de combinação das trocas de calor.

O conceito de temperatura equivalente foi introduzido inicialmente por Dufton (1932 apud NILSSON, 2004), que construiu um sensor especial constituído de um cilindro oco de cobre pintado de preto para medir a temperatura equivalente. Mais tarde, Bedford (1936 apud NILSSON, 2004) propôs uma expressão para o

\footnotetext{
${ }^{2}$ BOHN, M. et al. Evaluation of the Thermal Environment Intractor Cabs. In: INTERNATIONAL CONFERENCE ON ENVIRONMENTAL ERGONOMICS, 4., Austin, 1990. Proceedings... Austin, 1990

${ }^{3}$ SCHWARB, R.; CONRAD, W.; MAYER, E. Correlation Etween Objective and Subjective Measurements of Thermal Confort. EQUIV Report, Holtzkirchen, v.4, 1999.
} 
cálculo da temperatura equivalente, apresentada na Equação 1.

$$
T_{e q}=0,522 \cdot T_{a r}+0,478 \cdot \overline{T_{r}}-0,21 \sqrt{V_{a r}} \cdot\left(37,8-T_{a r}\right)
$$

Onde:

$\mathrm{T}_{\text {eq }}$ : temperatura equivalente $\left[{ }^{\circ} \mathrm{C}\right]$;

$\mathrm{V}_{\mathrm{ar}}$ : velocidade média do ar $[\mathrm{m} / \mathrm{s}]$;

$\bar{T}_{r}$ : temperatura radiante média $\left[{ }^{\circ} \mathrm{C}\right]$; e

$\mathrm{T}_{\mathrm{ar}}$ : temperatura do ar $\left[{ }^{\circ} \mathrm{C}\right]$.

Embora a Equação 1 descreva os efeitos da velocidade do ar, ela não leva em consideração o tipo de vestimenta usada, o que foi feito por Madsen, Olesen e Kristensen (1984), que desenvolveu a expressão apresentada na Equação 2 .

$$
T_{e q}=0,55 \cdot T_{a r}+0,45 \cdot \bar{T}_{r}+\frac{0,24-0,75 \cdot \sqrt{V_{a r}}}{1+I_{R}} \cdot\left(36,5-T_{a r}\right)
$$

nde:

$\mathrm{I}_{\mathrm{R}}$ : índice de isolamento total da roupa [clo].

Assim, com base no que foi apresentado, no presente trabalho são avaliadas as condições de conforto térmico em salas cirúrgicas utilizando o método de Fanger e temperaturas equivalentes.

\section{Método}

Para a realização do trabalho, primeiro foram levantadas as condições de conforto térmico com base no modelo de Fanger (1972) e, posteriormente, os valores de temperatura equivalente que representam a sensação térmica de conforto tanto para o presente trabalho quanto para os trabalhos de Wyon, Lidwell e Willians (1968) e Mora, English e Athienitis (2001).

\section{Avaliação de conforto térmico}

Com o objetivo de se avaliarem as condições de conforto térmico da equipe cirúrgica, realizaram-se medições das variáveis ambientais e aplicou-se um questionário. $\mathrm{O}$ questionário, baseado no trabalho de Mora, English e Athienitis (2001), foi aplicado ao término de cada cirurgia. Nesse questionário cada membro da equipe cirúrgica descreveu sua sensação térmica, seguindo a escala de sensações térmicas apresentada na Tabela 1 . Ao todo foram respondidos 120 questionários.

Para a avaliação das condições de conforto térmico para cada membro da equipe cirúrgica, foram analisados os valores que representam os votos de sensação térmica obtidos dos questionários e comparados com os valores de PMV obtidos pelo método de Fanger, com a utilização de valores de medição das variáveis ambientais - temperatura do ar, velocidade do ar, temperatura de globo (para determinação da temperatura radiante média) e umidade relativa do ar - e das variáveis pessoais isolamento térmico da roupa e metabolismo.

Estudaram-se quatro situações de movimentação do ar, função do sistema de ventilação, conforme apresentado na Tabela 2. O estudo de condições de conforto com o sistema de ventilação desligado foi possível de ser realizado em procedimentos cirúrgicos bastante simples, com risco de infecção extremamente baixo.

Avaliaram-se as condições de conforto térmico considerando quatro membros da equipe cirúrgica, cirurgião, instrumentador, anestesista e enfermeiro, que apresentam diferenças quanto ao isolamento térmico da vestimenta, o tipo de atividade que exerce e a posição em que se encontra na sala.

\begin{tabular}{l|l}
\hline Condições & \multicolumn{1}{c}{ Características do sistema de ventilação } \\
\hline Ensaio A & Sistema desligado. \\
\hline Ensaio B & $\begin{array}{l}\text { Sistema com insuflamento pela parede na parte superior e } \\
\text { retorno pela mesma parede, na parte inferior do lado oposto. }\end{array}$ \\
\hline Ensaio C & Sistema com insuflamento e retorno pelo teto. \\
\hline Ensaio D & Sistema com fluxo unidirecional (laminar). \\
\hline
\end{tabular}

Tabela 2 - Condições de ensaio 
A medição das variáveis ambientais foi realizada segundo procedimentos e métodos de medição apresentados na norma ISO 7726 (ISO, 1998). Os sensores foram dispostos ao longo da sala, e os dados foram coletados, em intervalos de $1 \mathrm{~min}$, com sistema de aquisição de dados.

Para a realização das medições, a sala cirúrgica foi dividida em duas zonas: a Zona 1, onde se encontram o paciente, 0 cirurgião e 0 instrumentador; e a Zona 2, onde se encontram o anestesista e o enfermeiro (Figura 1). Na região denominada de Zona 1 foram feitas, inicialmente, medições sem a ocorrência de cirurgias nas três condições estudadas. Foram medidas velocidades e temperaturas do ar em quatro alturas $(0,1 \mathrm{~m}, 0,6 \mathrm{~m}$, $1,1 \mathrm{~m}$ e 1,7 $\mathrm{m}$ do nível do piso, previstas na norma ASHRAE 55:2004) e temperatura de globo a $1 \mathrm{~m}$ do piso. Isso foi feito com o objetivo de se levantarem dados da movimentação do ar nessa região, pois durante a cirurgia era possível a colocação de apenas um sensor, de temperatura e umidade do ar, acima da mesa cirúrgica.

Na região denominada de Zona 2 foram realizadas medições durante as cirurgias de temperaturas e velocidades do ar, também nas quatro alturas previstas na norma ASHRAE 55:2004 e em quatro pontos diferentes ao longo da sala, totalizando 16 pontos. Além disso, foram realizadas medições de umidade do ar e temperatura de globo em um ponto a $1 \mathrm{~m}$ do piso.
Os sensores utilizados para as medições de temperatura foram termômetros de resistência; para as medições de velocidades, foram utilizados anemômetros omnidirecionais; e para a medição da umidade do ar, utilizou-se higrômetro. A Tabela 3 apresenta as características dos instrumentos de medição utilizados.

Para a avaliação das condições de conforto térmico utilizando o método de Fanger, é necessário estabelecer o valor do isolamento térmico total da roupa e o metabolismo para cada membro da equipe cirúrgica.

Devido ao fato de as vestimentas utilizadas em salas cirúrgicas serem relativamente padronizadas, foi possível utilizar valores de isolamento térmico de roupa apresentados em Mora, English e Athienitis (2001). Para os cirurgiões e instrumentadores, consideraram-se valores de isolamento total da roupa de $0,86 \mathrm{clo}$, e para enfermeiros e anestesistas, valores de 0,42 clo. $\mathrm{O}$ nível de atividade para os membros da equipe cirúrgica foi obtido da norma ASHRAE 55:2004, considerando atividades similares. Para os cirurgiões, foram considerados valores de 1,6 met, e para o restante da equipe, foram considerados valores de 1,4 met, valores também utilizados por Mora, English e Athienitis (2001). Mais detalhes do método e dos resultados apresentados a seguir podem ser encontrados em Felix (2008).
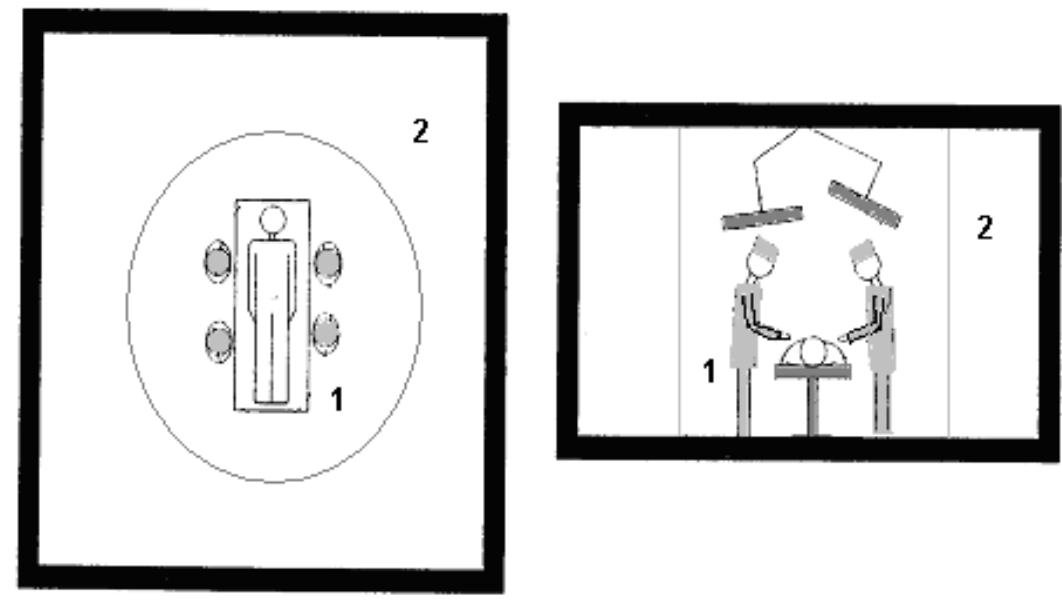

Fonte: Mora, English e Athienitis (2001).

Figura 1 - Vista em planta das regiões nas salas cirúrgicas

\begin{tabular}{c|c|c}
\hline Variáveis & Faixa de medição & Precisão \\
\hline Temperatura do ar & $0{ }^{\circ} \mathrm{C}$ a $50{ }^{\circ} \mathrm{C}$ & $\pm 0,3{ }^{\circ} \mathrm{C}$ \\
\hline Velocidade do ar & $0 \mathrm{~m} / \mathrm{s} \mathrm{a} 1 \mathrm{~m} / \mathrm{s}$ & $\pm(0,04+3 \%$ Var $)$ \\
\hline Umidade do ar & $20 \%$ a $90 \%$ & $\pm 5 \%$ \\
\hline
\end{tabular}

Tabela 3 - Características dos instrumentos utilizados 


\begin{tabular}{l|c|c|c|c}
\hline & Ensaio A & Ensaio B & Ensaio C & Ensaio D \\
\hline Temperatura do ar $\left(^{\circ} \mathbf{C}\right)$ & $26,0 \pm 0,4$ & $24,2 \pm 0,7$ & $27,1 \pm 1,0$ & $27,3 \pm 1,0$ \\
\hline Temperatura radiante média $\left({ }^{\circ} \mathbf{C}\right)$ & $29,0 \pm 0,4$ & $28,6 \pm 0,6$ & $29,5 \pm 0,5$ & $29,4 \pm 0,7$ \\
\hline Umidade relativa média do ar $(\boldsymbol{\%})$ & $45 \pm 8$ & $42 \pm 8$ & $37 \pm 6$ & $36 \pm 7$ \\
\hline Velocidade do ar $(\mathbf{m} / \mathbf{s})$ & $0,10 \pm 0,05$ & $0,20 \pm 0,07$ & $0,25 \pm 0,09$ & $0,20 \pm 0,06$ \\
\hline
\end{tabular}

Tabela 4 - Valores obtidos nos ensaios na Zona 1

\begin{tabular}{l|c|c|c|c}
\hline & Ensaio A & Ensaio B & Ensaio C & Ensaio D \\
\hline Temperatura do ar $\left({ }^{\circ} \mathbf{C}\right)$ & $23,5 \pm 0,6$ & $20,3 \pm 0,8$ & $22,9 \pm 0,4$ & $22,2 \pm 0,4$ \\
\hline Temperatura radiante média $\left({ }^{\circ} \mathbf{C}\right)$ & $25,6 \pm 0,4$ & $23,0 \pm 0,8$ & $24,1 \pm 0,5$ & $25,6 \pm 0,4$ \\
\hline Umidade relativa do ar $(\%)$ & $50 \pm 5$ & $48 \pm 8$ & $44 \pm 5$ & $45 \pm 5$ \\
\hline Velocidade do ar $(\mathbf{m} / \mathbf{s})$ & $0,10 \pm 0,05$ & $0,25 \pm 0,07$ & $0,30 \pm 0,08$ & $0,20 \pm 0,05$ \\
\hline Temperatura do ar no insuflamento $\left({ }^{\circ} \mathbf{C}\right)$ & -- & $15,6 \pm 0,5$ & $20,3 \pm 0,4$ & $21,7 \pm 0,5$ \\
\hline
\end{tabular}

Tabela 5 - Valores obtidos nos ensaios na Zona 2

\begin{tabular}{c|c|c|c}
\hline & Insuflamento/Zona 1 & Insuflamento/Zona 2 & Zona 1/Zona 2 \\
\hline Ensaio A & -- & - & 2,5 \\
\hline Ensaio B & 8,6 & 4,7 & 3,9 \\
\hline Ensaio C & 7,0 & 2,6 & 4,4 \\
\hline Ensaio D & 5,6 & 0,5 & 5,1 \\
\hline
\end{tabular}

Tabela 6 - Diferenças de temperaturas

\section{Análise de resultados}

Nas Tabelas 4 e 5 são apresentados valores de medição que caracterizam, respectivamente, o ambiente na Zona 1 e na Zona 2, para cada condição estudada. Não houve variação significativa das medidas ao longo do tempo, pois os ambientes são bastante homogêneos. As incertezas de medição apresentadas nas Tabelas 4 e 5 são o resultado da combinação das incertezas dos equipamentos e dos desvios padrão (COLEMAN; STEELE, 1989; INSTITUTO..., 1998) em cada condição de ensaio.

A Tabela 6 apresenta os valores das diferenças de temperaturas entre o insuflamento e as Zonas 1 e 2, e a diferença de temperatura entre as Zonas 1 e 2 para cada condição estudada. É possível verificar que o Ensaio D foi o que apresentou as menores diferenças de temperaturas entre o insuflamento e as Zonas 1 e 2 e as maiores diferenças de temperatura entre as Zonas 1 e 2.

\section{Condições de conforto térmico}

Nas Figuras 2 e 3 são apresentados, respectivamente, os valores de PMV calculados para as quatro condições estudadas utilizando os dados experimentais e o método de Fanger (FANGER, 1972; ISO, 2005) e os valores de voto de sensação térmica obtidos a partir do questionário aplicado a cada membro da equipe cirúrgica logo após as cirurgias.

É possível verificar que os valores de PMV obtidos com o método de Fanger (Figura 2) e os valores de voto de sensação térmica obtidos com o voto da equipe cirúrgica (Figura 3) apresentam uma mesma tendência, o que mostra a adequação da utilização do método de Fanger na avaliação de condições de conforto térmico também em salas cirúrgicas. As diferenças verificadas estão relacionadas, principalmente, com a dificuldade em se determinarem os valores de isolamento térmico da roupa e do nível de atividade.

Analisando as condições de conforto térmico a partir dos valores de PMV calculados (Figura 2), verifica-se que os cirurgiões e instrumentadores estariam desconfortáveis, com sensação de calor, em todas as situações analisadas. O nível de desconforto dos cirurgiões e instrumentadores seria praticamente o mesmo. Para os outros membros da equipe cirúrgica, isto é, os anestesistas e os enfermeiros, as melhores condições seriam as dos Ensaios A e D, pois estão dentro do intervalo considerado confortável, e as demais apresentaram condições levemente desconfortáveis. 


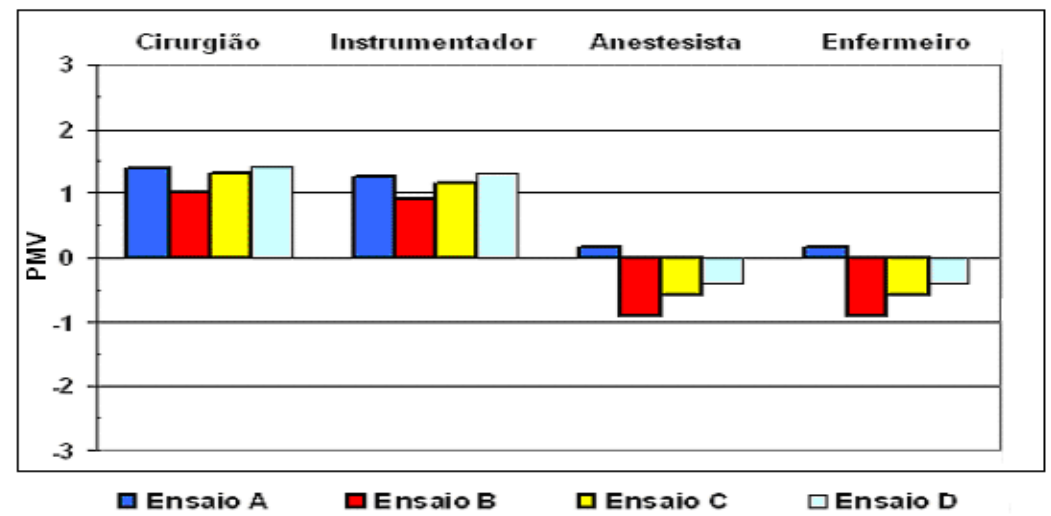

Figura 2 - Valores de PMV calculados pelo método de Fanger (1972)

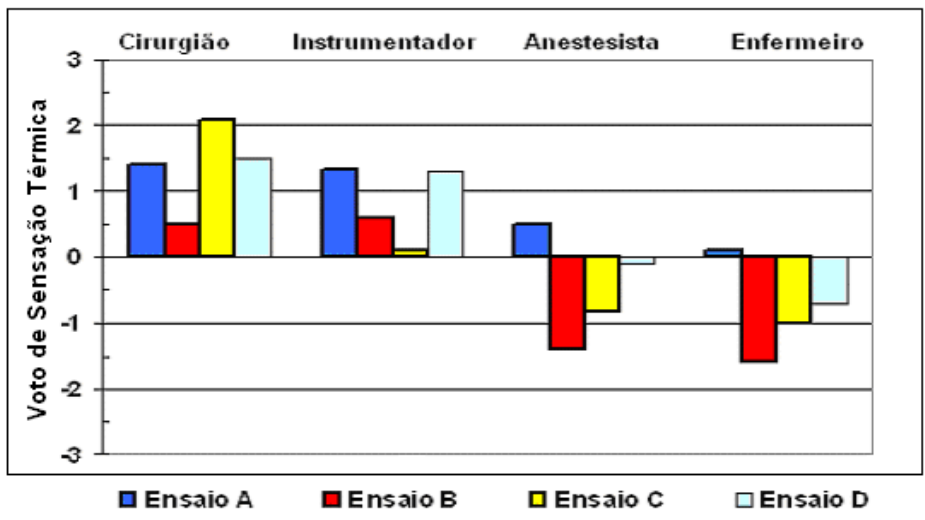

Figura 3 -Valores de voto de sensação térmica obtidos dos questionários aplicados após as cirurgias

\begin{tabular}{l|c|c|c|c}
\hline & Ensaio A & Ensaio B & Ensaio C & Ensaio D \\
\hline Temperatura do ar na Zona $1\left(^{\circ} \mathbf{C}\right)$ & 17,5 & 19,5 & 18,5 & 18,3 \\
\hline Temperatura do ar insuflado $\left({ }^{\circ} \mathbf{C}\right)$ & - & 11,0 & 11,5 & 13,0 \\
\hline $\begin{array}{l}\text { Isolamento térmico da roupa }(\mathbf{c l o}) \\
\text { (anestesistas e enfermeiros) }\end{array}$ & 0,73 & 0,86 & 0,86 & 0,86 \\
\hline
\end{tabular}

Tabela 7 - Valores ideais de temperatura do ar e de isolamentos térmicos da roupa

Na Tabela 7 são apresentados os valores de temperatura máxima do ar na Zona 1 , de temperatura de insuflamento do ar e de isolamento térmico mínimo da roupa de anestesistas e enfermeiros, que seriam necessários para que a equipe cirúrgica trabalhe em condições de conforto térmico. No cálculo das temperaturas máximas do ar na Zona 1, necessárias para os cirurgiões trabalharem em condições de conforto térmico, foram considerados os valores das variáveis ambientais na Zona 1 em cada ensaio velocidade do ar, umidade relativa do ar e temperatura radiante média -, bem como os valores referentes à vestimenta e ao metabolismo de cirurgiões, na determinação das temperaturas do ar que resultem em condições de conforto térmico, $\mathrm{PMV}=0$. Em seguida, com os valores obtidos da temperatura máxima do ar na Zona 1, e considerando as diferenças de temperatura entre as zonas (Tabela 6), foram obtidos os valores ideais de temperatura do ar de insuflamento e de temperatura máxima do ar na Zona 2 em cada ensaio. Finalmente, com os valores de temperatura máxima do ar na Zona 2 juntamente com os valores das variáveis ambientais na Zona 2 velocidade do ar, umidade relativa do ar e temperatura radiante média -, bem como os valores referentes ao metabolismo de anestesistas e enfermeiros, foi possível determinar o isolamento térmico mínimo da roupa de anestesistas e enfermeiros, também considerando as condições de conforto térmico de Fanger, PMV $=0$ (FANGER, 1972; ISO, 2005). 


\section{Estudo comparativo com resultados de outros trabalhos}

Na Tabela 8, são apresentados os valores utilizados para o cálculo de temperatura equivalente para cada membro da equipe cirúrgica, considerando os trabalhos de Wyon, Lidwell e Willians (1968), de Mora et al. (2001), e os valores do presente trabalho. Todos os valores de variáveis ambientais são médias dos valores obtidos em cada trabalho. No trabalho de Wyon, Lidwell e Willians (1968), os valores de isolamento térmico das roupas não foram apresentados, tendo sido adotados os mesmos valores do trabalho de Mora, English e Athienitis (2001) e do presente trabalho.

$\mathrm{Na}$ determinação de conforto das temperaturas do ar para os instrumentadores, anestesistas e enfermeiros (Tabela 8), foi realizado procedimento similar àquele utilizado na determinação das temperaturas de conforto dos cirurgiões (temperaturas máximas do ar na Zona 1, Tabela 7). Isto é, os valores das temperaturas do ar para esses membros da equipe cirúrgica também foram obtidos a partir dos valores das variáveis ambientais de velocidade do ar, umidade relativa do ar e temperatura radiante média em suas respectivas zonas de trabalho (Zonas 1 e 2), e das características pessoais de vestimenta e de nível de atividade de cada um desses membros da equipe cirúrgica, considerando condição de conforto térmico de Fanger, PMV = 0 (FANGER, 1972; ISO, 2005).
Utilizando a Equação 2, foram obtidos os valores de temperaturas equivalentes apresentados na Figura 4 e na Tabela 9. O Ensaio $A$ não foi considerado, pois foi realizado com o sistema de ar condicionado desligado, o que não é recomendado na maioria dos procedimentos cirúrgicos.

Pela análise da Figura 4 e da Tabela 9, verifica-se que a temperatura equivalente média de todos os resultados apresentados é de 21,9 oC, com desvio padrão de $\pm 0,6$ oC - dentro da faixa de incertezas de medição.

O resultado da análise mostra que nos três trabalhos chegou-se a valores praticamente iguais de temperaturas equivalentes de conforto térmico, em torno de $22 \mathrm{oC}$, para todos os membros da equipe cirúrgica.

Esse resultado é particularmente útil, pois permite, juntamente com a utilização da Equação 2, avaliar diferentes condições ambientais e pessoais de propiciar condições de conforto térmico aos diferentes membros da equipe cirúrgica.

\section{Conclusões}

Verificou-se que é difícil prover condições de conforto térmico para toda a equipe cirúrgica, principalmente devido a fatores pessoais, como o tipo de vestimenta e o nível de atividade. Por se tratar do profissional mais exigido da equipe cirúrgica, com maior grau de responsabilidade, o conforto térmico do cirurgião deve ser priorizado.

\begin{tabular}{|c|c|c|c|c|c|c|c|c|}
\hline & \multicolumn{4}{|c|}{ Cirurgião } & \multicolumn{4}{|c|}{ Instrumentador } \\
\hline & $\mathbf{T}_{\mathrm{ar}}\left({ }^{\mathbf{o}} \mathbf{C}\right)$ & $\mathbf{V}_{\mathrm{ar}}(\mathbf{m} / \mathbf{s})$ & $\mathbf{I}_{\mathbf{R}}($ clo $)$ & $\bar{T}_{r}\left({ }^{\mathbf{o}} \mathbf{C}\right)$ & $\mathbf{T}_{\mathrm{ar}}\left({ }^{\circ} \mathbf{C}\right)$ & $\mathbf{V}_{\mathrm{ar}}(\mathbf{m} / \mathbf{s})$ & $\mathbf{I}_{\mathbf{R}}($ clo $)$ & $\left.\bar{T}_{r} \quad{ }^{\mathbf{o}} \mathbf{C}\right)$ \\
\hline Wyon & 19,5 & 0,13 & 0,86 & 24,0 & 20,1 & 0,13 & 0,86 & 24,0 \\
\hline Mora & 19,0 & 0,25 & 0,86 & 27,0 & 19,0 & 0,25 & 0,86 & 27,0 \\
\hline Ensaio B & 19,5 & 0,20 & 0,86 & 28,6 & 19,0 & 0,20 & 0,86 & 28,6 \\
\hline Ensaio C & 18,5 & 0,25 & 0,86 & 29,5 & 19,0 & 0,25 & 0,86 & 29,5 \\
\hline \multirow[t]{3}{*}{ Ensaio D } & 18,3 & 0,20 & 0,86 & 29,4 & 19,0 & 0,20 & 0,86 & 29,4 \\
\hline & \multicolumn{4}{|c|}{ Anestesista } & \multicolumn{4}{|c|}{ Enfermeiro } \\
\hline & $\mathbf{T}_{\mathrm{ar}}\left({ }^{\mathrm{o}} \mathrm{C}\right)$ & $\mathrm{V}_{\mathrm{ar}}(\mathbf{m} / \mathbf{s})$ & $\mathbf{I}_{\mathbf{R}}(\mathbf{c l o})$ & $\bar{T}_{r}{ }_{\left({ }^{\mathbf{o}} \mathbf{C}\right)}$ & $\mathbf{T}_{\mathrm{ar}}\left({ }^{\circ} \mathbf{C}\right)$ & $\mathbf{V}_{\mathrm{ar}}(\mathbf{m} / \mathbf{s})$ & $\mathbf{I}_{\mathbf{R}}(\mathbf{c l o})$ & $\bar{T}_{r}\left({ }^{\circ} \mathbf{C}\right)$ \\
\hline Wyon & 21,5 & 0,13 & 0,42 & 24,0 & 19,5 & 0,13 & 0,42 & 24,0 \\
\hline Mora & 23,0 & 0,20 & 0,42 & 23,0 & 23,00 & 0,20 & 0,42 & 23,0 \\
\hline Ensaio B & 22,0 & 0,25 & 0,42 & 23,0 & 22,00 & 0,25 & 0,42 & 23,0 \\
\hline Ensaio C & 22,0 & 0,30 & 0,42 & 24,1 & 22,00 & 0,30 & 0,42 & 24,1 \\
\hline Ensaio D & 22,0 & 0,20 & 0,42 & 25,6 & 22,00 & 0,20 & 0,42 & 25,6 \\
\hline
\end{tabular}

Tabela 8 - Valores utilizados para os cálculos da temperatura equivalente 


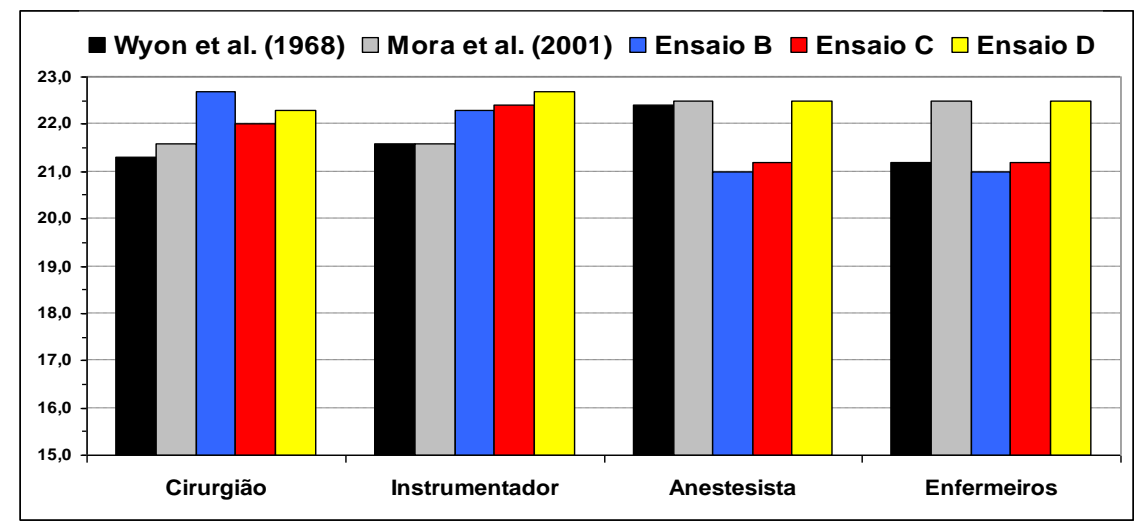

Figura 4 - Comparação de temperaturas equivalentes de conforto $\left({ }^{\circ} \mathrm{C}\right)$

\begin{tabular}{l|c|c|c|c}
\hline & Cirurgião & Instrumentador & Anestesista & Enfermeiro \\
\hline Wyon et al. (1968) & 21,3 & 21,6 & 22,4 & 21,2 \\
\hline Mora et al. (2001) & 21,6 & 21,6 & 22,5 & 22,5 \\
\hline Ensaio B & 22,7 & 22,3 & 21,0 & 21,0 \\
\hline Ensaio C & 22,0 & 22,4 & 21,2 & 21,2 \\
\hline Ensaio D & 22,3 & 22,7 & 22,5 & 22,5 \\
\hline
\end{tabular}

Tabela 9 - Quadro comparativo de temperaturas equivalentes de conforto $\left({ }^{\circ} \mathrm{C}\right)$

Os resultados obtidos também permitiram verificar que o sistema de fluxo unidirecional ("laminar") apresenta melhores condições de conforto térmico. Adicionalmente, esse sistema também apresenta maior potencial de controle de contaminantes no campo cirúrgico (Zona 1). Isso ocorre em função do tipo de movimentação do ar, com insuflamento de ar diretamente no campo cirúrgico, com menores diferenças de temperaturas ao longo da sala e maior eficiência na remoção de material particulado (PEREIRA, 2008).

Enfim, os resultados mostraram que há necessidade de cuidados redobrados quando se está tratando de climatização em salas cirúrgicas. Se, por um lado, o bem-estar da equipe cirúrgica é importante para o sucesso do procedimento cirúrgico, os riscos de contaminação aérea não podem ser negligenciados de forma alguma. Assim, a solução do problema deve considerar simultaneamente os efeitos da movimentação do ar na diminuição da possibilidade de contaminação aérea e na obtenção das melhores condições possíveis de conforto térmico.

Finalmente, resultados de estudo comparativo com trabalhos de outros autores mostraram que temperaturas equivalentes em torno de $22{ }^{\circ} \mathrm{C}$ correspondem a condições de conforto térmico para os diferentes membros da equipe cirúrgica. Tal resultado é particularmente útil na busca de se proverem condições de conforto térmico aos diferentes membros da equipe cirúrgica.

\section{Referências}

ASHRAE. Standard 55: thermal environmental conditions for human occupancy. Atlanta, 2004.

BEDFORD, T. The Warmth Factor in Comfort at Work. Rep. Industry Health Res, London, v. 76, 1936.

COLEMAN, H. W.; STEELE, W. G.

Experimentation and Uncertainty Analysis for Engineers. New York: John Wiley \& Son, 1989.

DHARA, S.; PITTET, D. Environmental Controls in Operating Theatres. Journal of Hospital Infection, v. 51, p. 79-84, jun. 2002.

DUFTON, A. F. The Equivalent Temperature of a Room and Its Measurement. Building Research Technical Paper, London, v. 13, 1932.

FANGER, P. O. Thermal Comfort: analysis and applications in environmental engineering. New York: McGraw-Hill, 1972.

FELIX, V. B. Condições de Conforto Térmico e de Desconforto Local em Salas Cirúrgicas.

2008. 80 f. Dissertação (Mestrado em Engenharia Mecânica ) - Escola Politécnica, Universidade de São Paulo, São Paulo, 2008.

HERMANS, R. D. Health Care Facility Design Manual: room design. In: ANNUAL MEETING ASHRAE TRANSACTIONS, 106., Minneapolis, 2000. Proceedings... Minneapolis, 2000. p. 162$167,2000$. 
INSTITUTO NACIONAL DE METROLOGIA, NORMALIZAÇÃO E QUALIDADE

INDUSTRIAL. Guia para Expressão da Incerteza de Medição. 2. ed. Rio de Janeiro: INMETRO; ABNT, 1998.

INTERNATIONAL ORGANIZATION FOR STANDARDIZATION. ISO 7726: thermal environments: instruments and methods for measuring physical quantities. Geneva, 1998.

INTERNATIONAL ORGANIZATION FOR STANDARDIZATION. ISO 7730: ergonomics of the thermal environment: analytical determination and interpretation of thermal comfort using calculation of the PMV and PPD indices at local thermal criteria. Geneva, 2005.

MADSEN, T. L.; OLESEN, B. W.; KRISTENSEN, N. K. Comparison Between Operative and Equivalent Temperature and Typical Indoor Conditions. ASHRAE Transactions, v. 90, p. 1077-1090, 1984.

MORA, R.; ENGLISH, M. J. M.; ATHIENITIS, A. K. Assessment of Thermal Comfort During Surgical Operations. ASHRAE Transactions, v. 107, n. 1, p. 52-62, 2001.

MOURA, D. Condições do Escoamento e de Conforto Térmico em Cabine de Aeronave. $74 \mathrm{f}$. 2009. Dissertação (Mestrado em Engenharia Mecânica ) - Escola Politécnica, Universidade de São Paulo, São Paulo, 2009.
NILSSON, H. O. Comfort Climate Evaluation with Thermal Manikin Methods and Computer Simulation Modes. 202 f. 2004. Thesis (Thesis) Department of Technology and Built Environment, University of Gaule, Sweden, Stockholm, 2004.

PEREIRA, M. L. Medição, Predição e Análise de Partículas Aéreas em Salas Cirúrgicas. 2008.

163 f. Tese (Doutorado em Engenharia Mecânica 1) - Escola Politécnica, Universidade de São Paulo, São Paulo, 2008.

PEREIRA, M. L.; TRIBESS, A. A Review of Air Distribution Patterns in Surgery Rooms Under Infection Control Focus. Engenharia Térmica, v. 4, n. 2, p. 113-121, out. 2005.

ROHLES JÚNIOR, F. H. Temperature \& Temperament: a psychologist looks at comfort. ASHRAE Journal, p. 14-22, feb. 2007.

SCHWAB, R.; CONRAD, W.; MAYER, E. Correlation Beetween Objective and Subjective Measurements of Thermal Comfort. EQUIV Report, Holtzkirchen, Germany, v. 4, 1999.

WYON, D. P.; LIDWELL, O. M.; WILLIANS, R. E. O. Thermal Comfort During Surgical Operations. Journal of Hygiene, v. 66, p. 229248, 1968.

\section{Agradecimentos}

Os autores Victor Barbosa Felix, Marcelo Luiz Pereira e Arlindo Tribess agradecem ao CNPq pela concessão de bolsa de mestrado, doutorado e de pesquisa respectivamente. Danilo de Moura agradece à FAPESP pela concessão de bolsa de mestrado. 